\title{
imGLAD: accurate detection and quantification of target organisms in metagenomes
}

Juan C Castro ${ }^{1,2}$, Luis M Rodriguez-R ${ }^{1,3}$ ， William T Harvey ${ }^{3}$, Michael R Weigand $^{3,4}$, Janet K Hatt ${ }^{3}$, Michell Q Carter ${ }^{5}$, Konstantinos T Konstantinidis ${ }^{\text {Corresp. } 1,2,3}$

${ }^{1}$ Center for Bioinformatics and Computational Genomics, Georgia Institute of Technology, Atlanta, Georgia, United States

2 School of Biological Sciences, Georgia Institute of Technology, Atlanta, Georgia, United States

3 School of Civil and Environmental Engineering, Georgia Institute of Technology, Atlanta, Georgia, United States

4 Division of Bacterial Diseases, Center for Disease Control and Prevention, Atlanta, Georgia, United States

5 Produce Safety and Microbiology, USDA-ARS Western Regional Research Center, U.S. Department of Agriculture, Albany, California, United States

Corresponding Author: Konstantinos T Konstantinidis

Email address: kostas@ce.gatech.edu

Accurate detection of target microbial species in metagenomic datasets from environmental samples remains limited because the limit of detection of current methods is typically inaccessible and the frequency of false-positives, resulting from inadequate identification of regions of the genome that are either too highly conserved to be diagnostic (e.g., rRNA genes) or prone to frequent horizontal genetic exchange (e.g., mobile elements) remains unknown. To overcome these limitations, we introduce imGLAD, which aims to detect (target) genomic sequences in metagenomic datasets. imGLAD achieves high accuracy because it uses the sequence-discrete population concept for discriminating between metagenomic reads originating from the target organism compared to reads from co-occurring close relatives, masks regions of the genome that are not informative using the MyTaxa engine, and models both the sequencing breadth and depth to determine relative abundance and limit of detection. We validated imGLAD by analyzing metagenomic datasets derived from spinach leaves inoculated with the enteric pathogen Escherichia coli 0157:H7 and showed that its limit of detection can be comparable to that of PCR-based approaches for these samples ( 1 cell/gram). 


\section{1 imGLAD: accurate detection and quantification of target organisms in}

\section{2 metagenomes}

3 Juan C. Castro ${ }^{1,2}$, Luis M. Rodriguez-R ${ }^{1,2,3}$, William T. Harvey ${ }^{3}$, Michael R. Weigand ${ }^{3+}$, Janet K. Hatt ${ }^{3}$,

4 Michell Q. Carter ${ }^{4}$, and Konstantinos T. Konstantinidis ${ }^{1,2,3+, *}$

$5{ }^{1}$ School of Biological Sciences, Georgia Institute of Technology, Atlanta, GA 30332, USA,

62 Center for Bioinformatics and Computational Genomics, Georgia Institute of Technology, Atlanta, GA

7 30332, USA,

$8{ }^{3}$ School of Civil and Environmental Engineering, Georgia Institute of Technology, Atlanta, GA 30332

9 USA,

$10{ }^{4}$ Produce Safety and Microbiology, USDA-ARS Western Regional Research Center, Albany, CA 94710 ,

11 USA and

12

$13{ }^{+}$Present Address: Michael R. Weigand, Division of Bacterial Diseases, Centers for Disease Control and

14 Prevention, Atlanta, GA 30329, USA

* To whom correspondence should be addressed.

Konstantinos T. Konstantinidis,

School of Civil \& Environmental Engineering,

Georgia Institute of Technology.

311 Ferst Drive, ES\&T Building, Room 3321,

Atlanta, GA, 30332.

Telephone: 404-639-4292

Email: kostas@ce.gatech.edu 


\section{ABSTRACT}

32 Accurate detection of target microbial species in metagenomic datasets from environmental samples 33 remains limited because the limit of detection of current methods is typically inaccessible and the 34 frequency of false-positives, resulting from inadequate identification of regions of the genome that are 35 either too highly conserved to be diagnostic (e.g., rRNA genes) or prone to frequent horizontal genetic 36 exchange (e.g., mobile elements) remains unknown. To overcome these limitations, we introduce 37 imGLAD, which aims to detect (target) genomic sequences in metagenomic datasets. imGLAD achieves 38 high accuracy because it uses the sequence-discrete population concept for discriminating between 39 metagenomic reads originating from the target organism compared to reads from co-occurring close 40 relatives, masks regions of the genome that are not informative using the MyTaxa engine, and models 41 both the sequencing breadth and depth to determine relative abundance and limit of detection. We 42 validated imGLAD by analyzing metagenomic datasets derived from spinach leaves inoculated with the 43 enteric pathogen Escherichia coli $\mathrm{O} 157: \mathrm{H} 7$ and showed that its limit of detection can be comparable to 44 that of PCR-based approaches for these samples ( 1 cell/gram). 


\section{INTRODUCTION}

48 Detection of target bacterial species and strains (e.g., pathogens) in environmental samples is a critical step for robust environmental, clinical and biodefense surveillance studies (Mande, Mohammed, and Ghosh 2012; Miller et al. 2013). A wide range of methods has been employed to target and monitor selected species in air, water, food or clinical samples. Traditional assays include microscopy, culturebased analyses and, in the case of pathogens, immunoassays that detect antigens expressed by the pathogen. However, these assays are typically cumbersome (e.g., results are available after at least 1-2 days), and cannot typically detect organisms that are resistant to cultivation or novel. Accordingly, cultureindependent techniques, including PCR-based amplification tests or sequencing of genomic DNA, have been developed more recently that provide more rapid and, often, more accurate means to diagnose and genotype bacterial species (Huang et al. 2017). Advances in sequencing technologies have also drastically improved DNA collection and sequencing from environmental samples. Currently, it is possible to collect DNA samples from the entire microbial community present in a sample, denoted as metagenomic datasets, which provides new opportunities for diagnostics. Nonetheless, several challenges remain to be addressed in order to qualify metagenomics as an everyday tool for the diagnostic laboratory. Due in part to these challenges, many clinical samples entering a public health laboratory remain undiagnosed for the causative agent despite being subjected to a battery of techniques (Miller et al. 2013).

Most importantly, assessment of the minimum amount of sequencing required for accurate detection of target bacterial species in a background of a complex microbial community remains challenging. This problem has important practical applications in environmental and clinical surveillance studies as keystone ecosystem organisms or pathogens may not be among the abundant taxa in situ. Detection limits vary depending on the sequencing effort and technology (e.g., read length), and the complexity of the microbial community sampled, i.e., the number and relative abundances of the species present in the sample and their relatedness to the target species. In most cases, these parameters or their effects on the limit of detection remain inaccessible. Experiments with increasing amounts of target DNA added to environmental samples have been performed in the past to empirically establish detection limits [e.g., (Be et al. 2013)]. However, a theoretical framework to establish limit of detection based on bioinformatics analysis of metagenomics is still lacking. Furthermore, such empirical approaches are typically cumbersome, and specific to the system tested.

Several methods to evaluate presence or absence of bacterial species based on best match or Bayesian analysis of read mapping patterns against a reference collection of genome sequences such as Pathoscope or Sigma (Ahn et al. 2015; Hong et al. 2014) have been recently developed. Additionally, taxonomic profilers such as MetaPhIAn (Segata et al. 2012; Truong et al. 2015) or MetaMLST (Zolfo et al. 2017) employ species- or strain-specific genetic markers to identify the different members of the community. However, these approaches rely on Single Nucleotide Polymorphism (SNP) pattern

83 differences against reference genes/genomes, which are difficult to robustly determine, especially in 
84

85

86

87

88

89

90

91

92

cases of low abundance (i.e., not enough reads available to reliably call SNPs). Importantly, no available tool can detect organisms that are not part of a reference genome database, and most tools are not easily adaptable to include new target genomes as references (e.g., the tools require re-computation of the typically large- training datasets or reference database to include new target organisms). Further, it is not clear how the co-presence of relatives of varying relatedness to the target organisms in the sample, as often is the case of environmental samples, affects detection ability and whether or not strain-level resolution can be achieved.

While these previous studies highlighted the challenges associated with accurate detection of genomes in metagenomic datasets, they also provided hints for possible solutions to the problem. More specifically, the detection problem can be framed as a classification problem based on two categories: a metagenomic dataset is designated as positive if the target genome is present; conversely, a negative dataset does not include any sequence originating from the target. Thus, training sets with positive and negative datasets could be used to train a classifier for reliable target detection. Likewise, the taxonomic profilers have shown that some regions of the genome can be used to reliably infer presence of a target, at the species or even sub-species (strain) levels, while other regions are not diagnostic enough. Therefore, the classification problem has two important parameters. One parameter is sequencing depth, i.e., how many times each base of the genome is sequenced or covered by sequencing reads, directly related to the relative abundance of the target organism in the sample and thus, the limit of detection. The other parameter is sequencing breadth, i.e., what fraction of the genome has to be sequenced, after removing (masking) regions of the genome that are not diagnostic enough, for reliable detection. By determining the minimum recovered fraction of the genome needed for detection, reliable detection can be established even in cases where species-specific genes are missing; for instance, due to incomplete sequencing or assembly of the target genome from the metagenomic dataset as an effect of low relative in-situ abundance.

Here, we present imGLAD (in-silico metagenomes for Genome Low-Abundance Detection), a new pipeline that incorporates a training classification step with positive and negative datasets as outlined above, and several computational optimizations to address the abovementioned limitations. Application of imGLAD to metagenomes derived from samples of known composition (mock) showed that it can reliably detect target organisms of interest in a background of closely related co-occurring relatives and frequently outperforms other methods.

\section{MATERIAL AND METHODS}

\section{Overview of the imGLAD pipeline}

imGLAD assumes that reads of a metagenomic dataset originate at random from all regions of the genome. Thus, the fraction of the genome that is recovered in the dataset (sequencing breadth) as well as the number of times each region is sequenced (sequencing depth), both depend on the abundance of 
121 the organism in the community. Highly conserved regions (e.g., rRNA and tRNA genes), as well as

122 regions resulting from recent horizontal gene transfer (e.g., transposase and integrase genes), can recruit

123 reads from other non-target genomes and misleadingly increase the value of sequencing depth (and

124 hence, estimated relative abundance) in some datasets depending on the gene composition of the

125 organisms present. To address this problem, we developed a framework to identify which fraction of a

126 target genome corresponds to reads that belong to the target and what fraction is the result of spurious

127 matches. This framework has two steps: initial training and subsequent prediction (Fig. 1). Training set

128 selection can be automatic or user defined. The automatic training generates reads from a randomly

129 selected number of genomes (default is 200 genomes) from RefSeq (Pruitt et al. 2007), and builds in-

130 silico-generated datasets of about 1 million reads each. Simulated reads from the target genome(s) are

131 then generated in a similar way and added to the former datasets in order to create the positive datasets

132 with decreasing target abundances. Reads from the target genome(s) are omitted for the construction of

133 negative datasets. All other genomes used to create the datasets are sampled in equal proportions (i.e.,

134 same relative abundances). The user can also choose the genomes to use to generate the training set

135 (e.g., genomes previously known to co-occur in the same environment). In this case, the construction of

136 the training set will be performed based on these genomes rather than the default genome collection from

137 RefSeq. Simulated Illumina-like reads are generated using ART-MountRainier (Huang et al. 2012) with

138 default settings. Simulation of reads from additional sequencing platforms is provided as an option, using

139 also ART-MountRainier. Reads from both positive and negative samples are then recruited against the

140 target genome sequence (reference) using BLAT (Kent 2002). Alternatively, BLAST can be used to

141 improve sensitivity at the expense of computational time (Altschul et al. 1997). By default, reads with

142 identity higher than $95 \%$ and at least $90 \%$ of the read length aligned are selected to calculate sequencing

143 breadth and sequencing depth, after normalizing for the size of the dataset. This level of identity has been

144 shown to capture well the genome-aggregate Average Nucleotide Identity (ANI) typically seen between

145 most currently named bacterial species, i.e., $>95 \%$ ANI within vs. $<95 \%$ ANI between species

146 (Konstantinidis \& Tiedje 2005; Rodriguez et al. 2018) and the sequence-discrete populations recovered

147 frequently in metagenomes of natural habitats (Caro-Quintero \& Konstantinidis 2012), although different

148 user-defined cut-offs can be used as well. Members of such sequence-discrete populations show high

149 gene-content and nucleotide sequence similarity among themselves, often -but not always- >95\% ANI,

150 and/or lower relatedness (e.g., $<90 \%$ ANI) to close relatives [reviewed in (Caro-Quintero \& Konstantinidis

151 2012)]. Sequencing depth (SD) is calculated as the number of reads mapping to the genome (N)

152 multiplied by the read length $(L)$ divided by the total length of the genome $(G)$, and sequencing breadth

$153(\mathrm{SB})$ is calculated as the number of bases covered $(\mathrm{B})$ divided by the total length of the genome, using

154 equations (1) and (2) below, respectively. If the genome consists of more than one contig (e.g., draft

155 genomes), the length is assumed to be the sum of the lengths of all contigs.

$156 \quad S D=L{ }^{*} N / G$

$157 \quad S B=B / G$ 
158

159

160

A logistic function is fitted to the resulting recruitment data (i.e., SB and SD values or SB values alone; see also below) that attempts to separate the positive from the negative training datasets in terms of sequencing depth and sequencing breadth (the latter two are the variables of the function). In particular, this approach calculates the parameters of the logistic function by computing the error in the training set, i.e., what SB and SD values are observed for the 100 positive vs. the 100 negative training datasets, and modifying the parameters accordingly to reduce the error until convergence is reached. Error is assessed by a log-likelihood maximization via gradient approach, which modifies the parameter values until the error is minimized. Regression coefficients of the logistic equation are calculated for the SD and SB variables as well as for an intercept term and thus, the model estimates three parameters, i.e., SD, SB, and intercept. Final parameters of the model are estimated by default only based on SB (sequencing breadth), as this variable was found to be the most discriminating parameter for positive vs. negative samples (see also below). However, an estimation including SD is also provided as an option in order to produce, in addition to the probability of presence/absence, an accurate estimation of the abundance of the target genome.

\section{Estimation of the probability of detection and limit of detection}

Once the parameters of the logistic function have been determined (above), SB and SD can be used to reliably predict the probability of presence of the target genome in any number of query metagenomes after the reads of the query have been recruited against the target genome and (observed) SB is estimated as described above for training datasets. The probability of presence is estimated according to:

$$
p=1-\frac{1}{1 \mp e^{-z}}
$$

Where $z$ is a linear function of the form $\beta^{T} t, \beta$ represents the regression parameters and $t$ is either a vector composed of the SD (Eq. 1) and SB (Eq. 2) or, by default, a one-dimensional variable corresponding to SB. Based on the model parameters (Eq. 3), it is possible to establish a detection limit for the target genome in each metagenomic dataset analyzed. This limit is defined as the minimum fraction (SB) that needs to be sampled in order to estimate a probability of presence at 0.95 . The result is displayed as a black solid line in a 2D plot of SB and SD (e.g., Fig. 2). The SD value observed based on the read recruitment, when corresponding to a probability value equal or higher to 0.95 , is then used to estimate the relative abundance of the organism in the sample. The SD corresponding to 0.95 probability then provides the limit of detection in terms of relative abundance.

\section{Filtering conserved regions}

To avoid spurious results from reads mapping on regions of the (target) genome with insufficient diversity (high sequence conservation such as rRNA genes) or frequently undergoing horizontal gene transfer such as mobile elements, the user can create a filter for these regions using MyTaxa (Luo et al. 2014a). This filter is created by predicting genes in the target genome and determining their classification weight using MyTaxa. If the MyTaxa classification score is at the bottom $5 \%$ or the gene is not scored (e.g., 
195

196

197

198

199

200

201

202

203

204

205

206

207

208

209

210

211

212

213

214

215

216

217

218

219

220

221

222

223

224

225

226

227

228

229

230

231

some hypothetical proteins) the gene is removed from the genome and further analysis. The filtered version of the genome is subsequently used for the model training and probability estimation steps.

\section{Bioinformatic tool comparisons and tool parameters used}

MetaPhIAn V2 (Truong et al. 2015) was run with the default settings using Bowtie version 2.2.8 (Langmead \& Salzberg 2012) for read mapping. MetaMLST (Zolfo et al. 2017) was used with default settings. PathoScope 2.0 (Hong et al. 2014) was run with default settings, using the same set of reference genomes that were used to build the training datasets for imGLAD.

Four tests were performed to assess specificity and sensitivity. In all cases, sensitivity was calculated as the proportion of properly classified positive datasets among the total number of positive datasets. Specificity was defined instead as the fraction of correctly identified negative datasets among all negative datasets examined. For the first test, metagenomic datasets were created with similar parameters to the training dataset of E. coli (i.e., 100 datasets from RefSeq genomes). These datasets were spiked with seven different concentrations of the $E$. coli genome in order to provide $1 \%$ to $7 \%$ coverage of the genome (i.e., sequencing breadth). In the second test, Human Microbiome Project (HMP) metagenomes were spiked with reads from the E. coli genome in order to provide $1 \%$ to $7 \%$ sequencing breadth as above. 571 HMP datasets were used for each $E$. coli concentration. In the third test, the datasets constructed in test 1 were spiked with reads from close relatives of E. coli, i.e., Klebsiella (81\% ANI), Salmonella (82\% ANI), and Escherichia fergusonii (92\% ANI), at random concentrations for each genome in addition to the $E$. coli reads. Finally, a test using close relatives, i.e., $>95 \%$ ANI representing strains of the same species, was performed in the HMP datasets in a similar way as described above for test \#3.

\section{Leaf inoculation experiments to test imGLAD and sample sequencing}

Fifty grams of field-grown spinach leaves were inoculated (spiked in) with cells of Escherichia coli O157:H7 strain RM6067, a strain linked to the 2006 spinach-associated outbreak in the U.S.A. (Carter et al. 2011). Three serial dilutions were performed resulting in three inoculation concentrations: $80,8 \times 10^{3}$ and $8 \times 10^{5}$ cells per pellet, plus a control sample with no inoculated cells. Cells for inoculation were obtained from single colonies that were grown overnight, and cell concentrations were determined by enumeration of colony-forming units (CFUs) on LB agar plates. Leaves were subsequently washed, the leaf wash was filtered to remove plant debris, and leaf-associated microorganisms were pelleted by centrifugation at $10,000 \mathrm{~g}$ for $10 \mathrm{~min}$ at $4^{\circ} \mathrm{C}$. DNA extraction was performed using MoBio UltraClean Microbial DNA isolation kit according to manufacturer's instruction (MoBio).

DNA sequencing libraries were prepared using the Illumina Nextera XT DNA library prep kit according to manufacturer's recommendations, except that the protocol was terminated after isolation of cleaned amplified double stranded libraries. Library concentrations were determined by fluorescent quantification using a Qubit HS DNA kit and Qubit 2.0 fluorometer (ThermoFisher Scientific, formerly Life Technologies) according to manufacturer's recommendations and libraries were run on a High-Sensitivity DNA chip 
232 using the Bioanalyzer 2100 instrument (Agilent) to determine average library insert sizes. An equimolar 233 mixture of the libraries (final loading concentration of $11 \mathrm{pM}$ ) was sequenced using a MiSeq reagent v3 kit 234 for 600 cycles ( $2 \times 300$ bp paired end run) on an in-house Illumina MiSeq instrument (Georgia Institute of 235 Technology), running the MiSeq control software v2.4.0.4 (MCS). Adapter trimming and demultiplexing of 236 sequenced samples was carried out by the MCS. Additionally, we used metagenomic datasets inoculated

237 with Bacillus anthracis DNA, which were made available previously (Be et al. 2013).

238

239

McFadden's pseudo- $R^{2}$ metric to assess the robustness of the logistic model/function with close relatives. The ability of the logistic model to distinguish between positive and negative training datasets when close relatives of increasing relatedness to the target genome were used in the training step was assessed using the McFadden's pseudo- $R^{2}$ metric. Specifically, the model determined (fitted) by imGLAD for a certain training dataset was compared to a standard, null logistic model which only contained an intercept variable. Effectively, this null model represented the standard logistic curve centered on the same point as the fitted imGLAD model but without any adjustment to the shape of that curve. Specifically, the metric was defined as:

$$
R_{\text {McFadden }}^{2}=1-\frac{\log \left(L_{C}\right)}{\log \left(L_{\text {null }}\right)}
$$

where $L_{C}$ is the maximized likelihood value for the fitted model and $L_{\text {null }}$ is the maximized likelihood value for the null model (intercept only, no covariates). Therefore, if the comparison shows perfect congruence between the two models (pseudo- $R^{2}$ close to 0 value) this means that the fitted model is not robust but similar to a randomly drawn model. In contrast, when pseudo- $\mathrm{R}^{2}$ approaches 1 , this denotes a robust fitted model. Note that pseudo- $R^{2}$ may not equal 1 , even for robust models, because the null model may approximate the fitted model estimated by imGLAD by chance alone in some iterations since it is drawn using the same intercept value. For this evaluation, the genome of one close relative at a time was added to the (negative and positive) training datasets at similar relative abundance (i.e., 10X, to ensured complete genome coverage) as the target genome ( $E$. coli strain $\mathrm{O} 157-\mathrm{H} 7$ ) was added in the positive datasets. The genomes of relatives were sorted into the following groups corresponding to their ANI values to the target genome (\%): 90, 95, 96, 97, 98.0, 98.2, 98.4, 98.6, 98.8, 99.0, 99.2, 99.4, 99.6, and 100. No genome was found with ANI value between $99.8 \%$ and $99.9 \%$ ANI. In addition to these related genomes, a uniform background dataset, which included 200 genomes showing $<80 \%$ ANI to E. coli strain $\mathrm{O} 157-\mathrm{H} 7$, was included to provide positive and negative training datasets of adequate complexity. Successive iterations of imGLAD with the resulting training datasets that each contained one close relative of varied $\mathrm{ANI}$ value to the target genome were performed, and models were evaluated using the equation (4) above as implemented in the scipy module of Python.

265

267 imGLAD is available through http://enve-omics.ce.gatech.edu/imGLAD/. Source code is available under

268 GNU General Public License v3.0 at https://github.com/jccastrog/imGLAD. imGLAD execution requires 
BLAT or BLAST to be installed, ART and the Python modules "scipy", "numpy", "screed", "statsmodels",

270 and "BioPython" (Jones et al. 2001; Oliphant 2006; Skipper \& Perktold 2010).

271

272 RESULTS

273 Training set for $E$. coli and $B$. anthracis

274 We evaluated imGLAD's performance on training datasets with E. coli strain O157:H7 EC4115, a strain

275

276

277

278

279

280

281

282

283

284

285

286

287

288

289

290

291

292

293

294

295

296

297

298

299

300

301

302

303

304

305 almost genetically identical to RM6067 used in the spinach inoculation experiments, i.e., $\sim 99.97 \%$ average nucleotide identity (or $\mathrm{ANI}$ ), and $B$. anthracis strain Ames as target genomes. The training datasets included closely related (but distinct) species of the same genus with ANI lower than 95\% (Fig. 1; see also below for within-species resolution), which corresponds to the frequently used standard for species demarcation (Goris et al. 2007) and encompass the sequence-discrete populations recovered frequently in metagenomes of natural habitats (Caro-Quintero \& Konstantinidis 2012). Although the predicted detection limit (from the training step) varied slightly for each of the two species, it was always possible to have confident detection (probability of presence $>99 \%$ ) when sequencing breadth was about 0.03 (or $3 \%$ of the total genome) or more based on the training datasets used (Fig. 2 \& Table 1). The model for $E$. coli was able to accurately separate positive from negative samples (probability of presence $>95 \%$ ) to a minimal value of sequencing breadth of 0.01 (Fig. 2A).

The logistic models from the training datasets were then applied to metagenomic datasets originating from environmental samples and spiked-in with the target genome (see Materials and Methods for details). For the E. coli experiment, 100 grams of field grown spinach leaves were inoculated (spiked in) with cells of strain RM6067, a strain linked to the 2006 spinach-associated outbreak in the U.S.A. (Carter et al. 2011). Three serial dilutions were performed resulting in three inoculation concentrations: $80,8 \mathrm{x}$ $10^{3}$ and $8 \times 10^{5}$ E. coli cells per spinach leaf microbiome (Table 1), plus a control sample with no inoculated cells (no cells were spiked in; although $E$. coli cells might have been present in the background leaf microbial community in low concentration). The resulting leaves were sequenced using the Illumina MiSeq short-read technology as described in the Material and Methods section. imGLAD was able to detect the target $E$. coli genome in all samples, even as low as 80 cells (Table 1). For the negative control, imGLAD provided values of sequencing breadth (0.007) and sequencing depth (0.042) that were consistent with the values of negative samples in the training set, i.e., the target genome was not present at the limit of detection of the approach (Fig. 2A; p-value for presence: 0.847 , Table 1). The matching reads in this case probably originated from natural $E$. coli populations present on the spinach leaves at low abundance or close relatives and/or spurious matches (Suppl Fig. 1).

The $B$. anthracis datasets were made previously available by Be and colleagues, and consisted of a soil microbial community DNA sample spiked with known quantities (genome equivalents) of DNA of $B$. anthracis strain Ames (Table 2), and sequenced using the Illumina GA-II technology (Be et al. 2013). A 
306 training set for $B$. anthracis was built in a similar way to the $E$. coli set; however, genomes that belong to

307 Bacillus cereus were excluded from the training dataset in this case as they show ANI values higher than

$30895 \%$ to $B$. anthracis. Based on the training datasets, a slightly higher limit of detection than the one for $E$.

309 coli was obtained (probability of presence $>95 \%$ ), with a minimum value of sequencing breadth of 0.039

310 (Fig. 2B). Among the 6 samples tested, a significant probability of presence ( $p>99 \%$ ) was obtained in

311 samples with 100 (3.8\% of the genome recovered), $1,000(56.2 \%), 10^{4}(98.3 \%)$, and $10^{5}(99.9 \%) B$.

312 anthracis genome equivalents. The samples with lower genome copy number (1 and 10 genomes) were

313 not identified as positive. Manual inspection of the number and position of matching reads to the $B$.

314 anthracis reference genome in the latter two datasets revealed about 2000 reads for the 10 genome copy

315 dataset and about 4000 reads for the 1 genome copy dataset, i.e., more reads were obtained with the

316 lower abundance dataset, indicating spurious matches (each dataset was on average 5.6 Gbp in size).

317 Further, the reads were concentrated in a few regions of the genome (not randomly distributed), which

318 was indistinguishable from negative datasets (Table 2, Fig. 2B, and Suppl Fig. 2). Thus, it appears that

319 the $B$. anthracis genomes might not have been sequenced adequately in the low copy number datasets.

320 This interpretation is also consistent with the fact that Be and colleagues employed a phi29-based, whole-

321 DNA amplification method that may have resulted in biased sequencing (e.g. only a few, not enough

322 diagnostic regions of the genome were amplified and sequenced), especially in the low $B$. anthracis

323 genome concentrations. Further, our results are also consistent with estimates that $100 \mathrm{Gbp}$ or more are

324 required to cover the complete genome diversity within typical soil microbial communities as described

325 previously (Luo et al. 2014b) and the conclusions of the original study by Be and colleagues.

326

327 Comparison to other tools

328 We compared the performance of imGLAD with other available platforms that can be used to identify the 329 taxa present in the sample. It should be pointed out, however, that these tools do not target a specific

330 organism/genome of interest but instead assess the total microbial community composition and thus, their

331 objective is slightly different than imGLAD's. Nonetheless, we were able to obtain meaningful results by

332 comparing imGLAD with popular tools for these purposes such as MetaPhIAn, MetaMLST, and

333 Pathoscope, which illustrated the advantages of imGLAD. In the $E$. coli and $B$. anthracis metagenomes

334 described above, imGLAD provided higher sensitivity than other tools, especially at low levels of

335 sequencing breadth. i.e., the proportion of properly classified positive datasets among the total number of

336 positive datasets. For instance, with $2 \%$ of the genome covered by sequencing reads in training datasets,

337 imGLAD accurately classified as positive 95\% of the datasets, whereas Pathoscope and MetaPhIAn

338 classified only about $47 \%$ and $16 \%$ of the datasets, respectively. Only when sampling $7 \%$ of the genome

339 or more, did these tools yield similar results to imGLAD (Fig. 3A). It should be noted that $7 \%$ is more than

340 twice the genome breadth (i.e., $3 \%$ of the genome) that imGLAD required to reach $100 \%$ classification

341 sensitivity. Comparisons against available tools that require higher target abundance to make confident

342 calls such as ConStrains (Luo et al. 2015) were not attempted as this would have been an unfair 
343 comparison. A few other tools such as GOTTCHA (Freitas et al. 2015) were proven to be too sensitive,

344 e.g., (positively) detecting E. coli even in E. coli negative training datasets, and thus, were not evaluated

345 further.

346

347 Additionally, we used a set of 571 metagenomic datasets of the HMP (http://www.hmpdacc.org/), in which

348 different concentrations of $E$. coli (target organism) reads were spiked to further test the specificity of 349 imGLAD against a naturally occurring background community (as opposed to in-silico generated 350 datasets) (Fig. 3B). These datasets were selected because they did not have any detectable amounts of 351 E. coli by any of the three tools to confound results. MetaPhIAn, which is optimized for human-associated 352 microbial communities, had better performance when tested against these HMP datasets relative to the 353 E. coli or B. anthracis datasets mentioned above. However, MetaPhIAn still required at least $5 \%$ of the 354 genome to be recovered in order to provide high confidence (positive) detection whereas imGLAD 355 achieved similar confidence with only $3 \%$ of the genome. Hence, imGLAD's sensitivity was superior, 356 especially in cases of low abundance of the target genome(s).

357

358 Improved detection was also observed in in-silico synthesized datasets that included close relatives (ANI 359 greater than $95 \%$ up to $98 \%$ compared to the target; for $>98 \% \mathrm{ANI}$, see below), although a larger fraction 360 of the genome was typically required in these cases $(\sim 7 \%)$ in order to achieve high specificity and 361 sensitivity by imGLAD. PathoScope and MetaPhIAn required an even higher fraction (at least 10\%) of the 362 target genome for comparable specificity and sensitivity (Fig. 3C; Suppl Fig. 3 shows similar results but 363 the background metagenome was from HMP instead of the in-silico synthesized datasets). In all cases 364 imGLAD achieved high specificity (>97\%), i.e., the fraction of correctly identified negative datasets among 365 all negative datasets examined (Suppl Fig. 4). In comparison, the other three tools never reached specificity higher than $90 \%$ on the same four tests (Suppl Fig. 4).

\section{Filtering of conserved regions}

369

In addition to creating a model using the whole genome, regions of the genome that provide a less reliable phylogenetic signal (e.g., regions that are highly conserved or contain mobile elements; see Material and Methods for details) can be identified by MyTaxa and removed/masked so that the prediction and/or the training steps can be repeated with the filtered genome for more accurate results. Briefly, MyTaxa was used to examine the classification accuracy of all genes in the genome as described previously (Luo et al. 2014a), and genes with classification score at the bottom 5\% or not scored (e.g., some hypothetical proteins) were removed (filtered out) from the genome, providing the filtered genome, which was used for the training step and further analysis. Filtering in general, improved the detection limit because reads mapping on masked regions were not counted (Fig. 4). For instance, filtering lowered the

378 minimum sequencing depth required for robust detection from $0.123 \mathrm{X}$ (no filtering applied) to $0.061 \mathrm{X}$ in the training datasets for $E$. coli (same p-value was used in both cases, equal to 0.05 ). The reduction in 
380

381

382

383

384

385

386

387

388

389

390

391

392

393

394

395

396

397

398

399

400

401

402

403

404

405

406

407

408

409

410

411

412

413

414

415

416

sequencing breadth however was not as dramatic as sequencing depth (e.g., 0.014 to 0.009 fraction of the total genome for the same datasets). The larger effect of filtering on sequencing depth than breadth was presumably attributable to the fact that filtering typically removed only a small part of the target genome (i.e., $<5 \%$ by default settings) that recruited a disproportionally high number of reads encoding highly conserved or frequently transferred genes. This interpretation is also consistent with the sigmoidal relationship between sequencing depth and breadth, which tends to flatten at high values of sequencing depth and becomes linear at lower values (Wendl et al. 2012). Hence, filtering with MyTaxa is recommended, in general, provided the target organism is represented in the database.

\section{Effect of relatedness of co-occurring genomes and strain-level resolution}

When building the training set, the user is able to add any non-target genomes that could be relevant for optimizing detection of the target genome such as genomes that are known to be present and relatively abundant in the sample or closely related species that should not contribute positive signal (i.e., reads mapping on shared regions of the genome). In general, imGLAD's sequencing breadth and/or depth for positive detection (i.e., the detection limit) was expected to be higher with higher relatedness of the nontarget genomes in the training set to the target genome. For instance, we tested different training sets that included relatives at different levels of ANI to the target genome ranging from 80 to almost $100 \%$ ANI. Consistent with our expectations, higher sequencing depth and breadth were required for robust detection when relatives showing 95 - $98.5 \%$ ANI (within species resolution) to the target co-occurred in the training dataset compared to relatives showing $90 \%$ or $80 \%$ ANI (between species resolution). This was due to the fact that more conserved and/or identical regions were present in the genome of the former relative to the latter. In fact, when co-occurring relatives were members of different species than the target species (i.e., show $<95 \%$ ANI), imGLAD's limit of detection was very similar to that of the training datasets without close relatives, i.e., $3 \%$ of the genome needed to be recovered for confident detection in most cases. When genomes of the same species were present (i.e., between 95 and $\sim 98.5 \%$ ANI), about 10\% of the genome was required, depending on the exact genomes considered and their relatedness (Fig. $3 C)$.

When relatives showed higher than $\sim 98.5 \%$ ANI (i.e., represented very closely related strains), imGLAD's detection efficiency was increasingly lower up to about $100 \%$, where reliable detection calls were often not possible. For instance, using McFadden's pseudo- $R^{2}$ metric to assess the ability of the logistic model to distinguish between positive and negative datasets, we found that the model parameters were reasonably well estimated $\left(R^{2}>0.7\right)$ until relatives showing about $98.5 \%$ ANI relatedness to the target were included in the training datasets, at which point the pseudo- $\mathrm{R}^{2}$ fell near 0.5 . Increasing the ANI relatedness of the (non-target) relatives further produced pseudo- $\mathrm{R}^{2}$ values either equal to or below 0.5 , indicating that the logistic model was not robust in identifying presence of the target in these cases (Fig. 5). Furthermore, as the relatedness increased, the contribution of the sequencing depth metric became less important. In fact, in some training datasets where close relatives with high identity to the 
417 target genome (98\% ANI or higher) were present and in relative high abundance, the estimated 418 parameters showed high variation during the training step. This resulted, for instance, in a positive slope 419 between sequencing breadth and depth, which was not reliable for estimating relative abundance and 420 detection limit, consistent with the McFadden's pseudo- $R^{2}$ statistics mentioned above. In these cases, 421 sequencing breadth alone represented a more reliable parameter for robust detection calls. It should be 422 noted, however, that the McFadden's pseudo- $\mathrm{R}^{2}$ evaluation showed that the model based on both 423 sequencing breadth and depth performed better than the one based on sequencing breadth alone (Fig. 424 5). This was due to the fact that the genome of close relatives was added in the training datasets at the 425 same abundances as the target genome for this evaluation, and that two parameters are typically better 426 than one for model fitting. Hence, the abundance of the target genome relative to that of the close relatives may affect the significance level of the sequencing depth for the logistic function, and the estimation of abundance of the target genome in a query metagenome.

429

In summary, gene-content differences among the target genome and the co-occurring, non-target close relatives become increasingly more important for robust detection in cases where the non-target genome(s) show increasing genetic relatedness to the target up to about $98.5 \% \mathrm{ANI}$, which marks the upper limit of relatedness of the non-target to the target genome for reliable selective detection of the 434 target. Within species (i.e., strain-level) resolution was achievable by imGLAD in such cases, but not when strains shared $>98.5 \%$ ANI. In the latter cases, the gene-content differences between target and non-target genomes, reflected on sequencing breadth in negative training datasets, was too small to resolve robustly. However, it is important to point out that, in practice, resolution among genomes sharing $>98.5 \% \mathrm{ANI}$ is typically not necessary for most applications since such genomes are typically members of the same sub-clade within species and share highly similar phenotypes, while resolving such closely related genomes would probably require detailed phylogenetic SNP analysis. Hence, training with close relatives is important for more stringent results when needed, especially in cases that the close relatives are known or highly anticipated to co-occur in the same samples with the target organism. When close relatives are not a concern, the default settings of imGLAD should be robust. The default settings are: to create 200 training examples (100 positive, 100 negative) with 1,000,000 simulated 150 bp Illumina reads per dataset; the reads are generated evenly from 200 genomes randomly sampled from NCBI; the positive datasets have additional reads from varying abundances of the target genome; the reads from each dataset are then aligned against the target genome using Blat with thresholds of $95 \%$ identity and $90 \%$ alignment length in order to build the logistic model.

449

\section{DISCUSSION}

451 We presented imGLAD, a novel algorithm that utilizes a logistic model-based learning approach for 452 accurate detection of target bacterial species in complex metagenomes, and for establishing detection 453 limits in a target species- and microbial community-specific manner. By building and analyzing training 
454

455

456

457

458

459

460

461

462

463

464

465

466

467

468

469

470

471

472

473

474

475

476

477

478

479

480

481

482

483

484

485

486

487

488

489

490

datasets with decreasing abundances of spiked-in reads originating from the target genome, imGLAD allows for highly reliable calls, while reducing the number of false positives (Fig. 2). Further, and contrary to available tools, imGLAD allows for reliable estimation of the detection limit of the metagenomic sequencing effort applied based on the training datasets of decreasing target genome abundance and a linear combination of both genome sequencing depth and genome sequencing breadth, or only sequencing breadth. The degree of sequence conservation of the genes of the target genome and their extent of horizontal gene transfer are also taken into account in estimating the limit of detection, which represents a substantial advantage over existing tools in minimizing false-positive calls. The results using both simulated datasets (e.g., Fig. 2) as well as experimental metagenomes (Tables 1 and 2) highlighted these advantages of imGLAD. However, imGLAD is not designed to detect all species present in a sample. Thus, it differs from taxonomic profiling software, and is computationally more expensive, due to the training step, if the goal is to detect more than a couple of targets. Rather, the goal of imGLAD is to provide highly accurate detection of specific, user-provided target species (e.g., pathogens or keystone species), including newly sequenced genomes. Further, imGLAD's logistic model, while computationally demanding to create (e.g., building in-silico training datasets), needs to be built only once and can subsequently be used multiple times with different metagenomes. This way, imGLAD could be used to efficiently and rapidly detect several target organisms in an environmental sample (by building a model for each target in advance). For instance, the modeling step typically took 8-12 hours on a node with 4 CPUs (2.5Mzh, $12 \mathrm{~Gb}$ memory), whereas the detection (i.e., making a call) step with one query metagenome took 0.5-1 seconds (once the read recruitment against the target genome was completed; the time required for the latter step varied, depending on genome and metagenome sizes).

A distinguishing strength of imGLAD is the detection of low abundance target genomes. Current tools for metagenomic profiling use specific markers or SNP patterns to identify and classify the species present in the sample [e.g., (Hong et al. 2014; Segata et al. 2012)]. However, at low levels of abundance, these markers may not be found, and SNPs cannot be called, and in some cases, the SNPs are called incorrectly such as in the case of MetaMLST (Zolfo et al. 2017), which requires high abundances (above $2 \mathrm{X}$ ) to make confident calls and thus, performed poorly in the tests we conducted compared to other tools or imGLAD (e.g., Fig. 3). Our approach is not focused on a particular region of the genome, but instead takes into account the whole genomic context. This provides higher recall while preserving precision (Fig. 3 \& Suppl Fig. 4). Further, methods based on read assignment depend on the comprehensiveness of their reference database and do not provide high precision when challenged with samples containing closely-related species (Hong et al. 2014). Accordingly, the tools evaluated here provided high false positive rates in such cases (Suppl Fig. 4), which can be concerning, for instance, in pathogen surveillance studies and environmental samples, where closely related strains of the same species may co-occur. imGLAD can provide reliable prediction even in such cases, although at the expense of a lower detection limit, assuming the close relatives are known and available and, hence, can be used as part of the training step as exemplified in the E. coli case above. However, if the query metagenome(s) include 
491

496

497

498

499

500

501

502

503

504

505

506

507

508

509

510

511

512

513

514

515

516

517

518

519

520

521

522

523

524

525

526

527

relatively abundant, non-target genomes more related to the target genome than any of the genomes used to construct the training datasets, then the predictions of imGLAD (or other tools) might not be highly accurate. In such cases, the user needs to recover the genome sequences of the relatives from the metagenome(s) using genome binning techniques, if the representative sequences are not available otherwise, in order to include them in the training dataset. The results presented here (e.g., Fig. 3A \& 5) provide a quantitative picture of this issue and its consequences on the accuracy of imGLAD as well as other tools.

Electrochemical immunoassays have shown promise in detecting pathogens such as $B$. anthracis or their toxins and can sometimes offer strain-level resolution. The limit of detection of these techniques can, in some cases, be $\sim 1 \mathrm{pg} / \mathrm{ml}$ (Sharma et al. 2016), which is below the limit of detection of imGLAD (56 $\mathrm{pg} / \mathrm{ml}-560 \mathrm{pg} / \mathrm{ml}$ corresponding to $10-100$ cells, respectively) based on the $E$. coli spike in experiment on spinach and current best practices for metagenomic sequencing and the samples analyzed here. Thus, immunoassays and culture-based approaches are still more sensitive than metagenomics, at least for highly complex metagenomes such as those of soils (but probably not as much for food and agricultural samples, the human gut or habitats of similar complexity), and could be used in combination with tools like imGLAD for more reliable and comprehensive results. A key advantage of imGLAD is that it has high specificity, which sometimes cannot be achieved by immunoassays or culture-based approaches. It should be noted that imGLAD might be able to offer resolution within species as well, e.g., by including in the training dataset genomes that are members of the same species but show sequence divergence from the target genome higher than that of the sequencing errors (e.g., 99\% ANI or less) and/or have substantial gene content differences (which can be captured by the sequencing breadth parameter). Subspecies resolution can also be obtained by analyzing the reads identified by imGLAD as representing the target genome in the query metagenome for their SNP pattern against a collection of genomes related to the target genome, using -for instance- the PathoScope approach (Hong et al. 2014) or a (manual) phylogenetic analysis of the reads.

Notably, imGLAD allows one to include new target genomes, including draft assemblies, in the training datasets, with little effort, which may be important for practical applications. Thus, the training step of imGLAD can be optimized with specific targets or habitats in mind such as the human gut and provide comparable, if not better results than tools that are already optimized for these communities. In contrast, most tools available require time and CPU-intensive updates of their reference databases to include new targets. Similarly, imGLAD can be easily optimized for different sequencing technologies as long as the training datasets are produced with reads simulating these technologies. This flexibility of imGLAD is an important advantage because the tenet "one approach fits all" does not apply well in the case of microbial detection in environmental samples, which are typically characterized by different degrees of co-occurring (non-target) relatives and are often sequenced based on different strategies nowadays.

It is also important to note that imGLAD's training step can be optimized to evaluate samples of different microbial community complexity, in addition to co-occurring relatives of varied genetic similarity 
528 to the target organism and different target genomes. For instance, more complex communities can be 529 simulated in the training step by including a higher number of different genomes in the training datasets 530 (200 genomes by default) and/or with different species abundance distributions, e.g., power law as 531 opposed to equal abundances (default setting). We have also found that training datasets with 200 532 genomes work well for most natural communities of medium-to-high complexity while increasing the 533 number of genomes only marginally increased the specificity or sensitivity of imGLAD (Suppl Fig. 5 \& 6), 534 in general, especially given the extra computational time required. Specifically, our assessment showed 535 that if the richness of the targeted microbial community (i.e., number of $95 \%$ ANI defined species or 536 clusters) is within one order of magnitude of the number of genomes used in the training (i.e., 1 through 5372000 species, for 200 genomes in the training datasets), the estimated imGLAD models are robust. 538 Hence, the default number of genomes $(n=200)$ should work for most microbial communities, and smaller 539 number of genomes could be used for less complex communities (e.g., $n=100$ ). The choice of which 540 genomes to use in the training dataset, in addition to just the number of genomes, is apparently also very 541 important for imGLAD's accuracy, e.g., inclusion or not of close relatives as mentioned above (e.g. Fig. 542 5). Analyzing the target metagenome with profiling tools such as MetaPhIAn and MyTaxa (Luo et al. 543 2014a; Truong et al. 2015) in advance can provide the end user with pertinent information on the 544 taxonomic composition of the target community. This information can guide the selection of the genomes 545 used for the training datasets so that close relatives, when present in the metagenome, can be included for more robust results (e.g., Fig. 5).

We also tested the effect of the size of the training datasets (i.e., library size), in the range of 100 to $1,000,000$ reads per dataset, on imGLAD's limit of detection (defined at the $p$ value $=0.05$ level as described above). As expected, larger training sets increased the limit of detection because the number of reads recruited by the target genome in such negative datasets (i.e., spurious matches or matches for poor phylogenetic marker genes) increased. However, we found that the increase was only minor overall and, in fact, leveled off around 0.03 sequencing breadth (3\%) for 100,000-1,000,000 reads/dataset (Suppl Fig. 7). Therefore, the default setting for training dataset $(1,000,000$ reads) should be robust for most applications. Additionally, we evaluated the accuracy of imGLAD detection calls using the receiver operating characteristic $(\mathrm{ROC})$ curves to measure positive rates and false negative rates over different sequencing breadth. It is important to note that, because the separation of the positive and negative datasets is very sharp (e.g., Fig. 2), the ROC curves were almost perfect for all cases where close relatives of $98 \%$ or less $\mathrm{ANI}$ to the target, or no close relatives, were present in the sample. In the datasets with close relative showing $98-99 \%$ ANI to the target being present, we found some overlap of the positive and negative datasets (Suppl Fig. 8), consistent with the results reported above for increasingly more challenging detection with relatives in the $98.5-100 \% \mathrm{ANI}$ range.

562 The decreasing costs of sequencing as well as technological improvements in sequencing throughput 563 and read length make it possible to use metagenomics to track specific bacterial populations in time 564 series data or monitor the presence of pathogens in clinical or environmental samples. As the number of 
565 studies with a focus on metagenomic datasets continue to increase, the need for fast, reliable and flexible

566 bioinformatics analysis tools to detect and characterize target populations will also continue to grow,

567 particularly in cases where isolation is not possible or is expensive. imGLAD represents an effective way

568 to accomplish this objective and to robustly evaluate the limitations of the underlying sequencing

569 technology or effort. imGLAD's default settings should work for most target microbial communities and

570 genomes, and the results presented here represent a guide for further optimization depending on the

571 specific goals of the study and the samples analyzed. Therefore, we anticipate that imGLAD will find

572 applications across the fields of clinical and environmental microbiology.

573

574 SOFTARE AND DATA AVAILABILITY

575 imGLAD is open source software available in the GitHub repository

576 (https://github.com/jccastrog/imGLAD). Spinach leaf metagenomes have been deposited in NCBI's SRA

577 database under accession number PRJNA432668.

578 
579 FIGURE LEGENDS

580 Figure 1. Schematic representation of imGLAD's pipeline. imGLAD has two main components. The

581 first part (training) consists of a learning procedure, in which a set of in-silico generated datasets are fitted

582 through a logistic model that aims to separate positive from negative datasets. For this, a database of 200

583 genomes is used to generate the simulated Illumina reads of these datasets. Reads simulated from the

584 target genome are then incorporated into half of the simulated datasets. The resulting datasets are

585 marked as positive for training while the other half is marked as negative. Sequencing depth and breadth

586 of the target (reference) genome are calculated for each dataset. A logistic function is then fitted to the

587 data to separate positive from negative examples. The regression parameters are stored for further use.

588 The second part (estimation) consists of estimating the sequencing breadth and/or depth values of the

589 target genome provided by the (recruited) reads of the experimental metagenomes, and comparison of

590 the derived sequencing depth and breadth values to those of the logistic function from the training step.

591

592 Figure 2. Identification of target genomes in metagenomic datasets with imGLAD. Positive datasets

593 (crosses) are separated from negative datasets (dots) through a logistic function (solid line) based on in-

594 silico training datasets. (A) Datasets with reads of $E$. coli are separated from negative datasets. (B)

595 Datasets with reads of $B$. anthracis are separated from negative datasets. Red asterisks denote the

596 position of the experimental metagenomes (remaining dots represent in-silico generated datasets); note

597 that the red star at the bottom of panel A denotes the negative control sample. Dashed lines represent the

598 sequence depth detection limit estimated by imGLAD. Note the differences in scale on the $x$-axes

599 between positive and negative datasets.

600

601 Figure 3. Performance of imGLAD in comparison to Pathoscope, MetaPhIAn and MetaMLST. (A)

602 in-silico synthesized datasets from 200 RefSeq genomes were spiked with E. coli EC11 reads at different

603 abundances (reflected by sequencing breadth, $x$-axes) to test the sensitivity of imGLAD (y-axes), i.e., the

604 proportion of properly classified positive datasets among the total number of positive datasets. (B) Similar

605 comparisons based on 571 datasets from the HMP project, which did not contain any E. coli signal and

606 were spiked with different concentrations of E. coli EC11 reads. (C) imGLAD was evaluated in the same

607 datasets as in panel A but this time the datasets included, in addition to the RefSeq genomes, $10 \mathrm{E}$. coli

608 genomes with ANI ranging between $95-98 \%$ to the target E. coli EC11 genome spiked in at the same

609 concentration (i.e., $0.3 \mathrm{X}$ ). Note that as sequencing breadth increases the sensitivity of the prediction is

610 higher for all tools tested, with the exception of MetaMLST that requires at least $2 \mathrm{X}$ sequencing depth for

611 robust detection (see text for details). However, imGLAD can effectively classify samples at $100 \%$

612 sensitivity (as positive samples in this case) with a sequencing breadth as low as 0.03 (i.e., $3 \%$ of the

613 target genome recovered) or less, whereas the other tools show lower sensitivity at these levels in all

614 cases evaluated.

615 
616 Figure 4. Effect of filtering of less informative genes by MyTaxa on minimum sequencing breadth

617 and depth. Genome regions of the E. coli target genome were classified by MyTaxa, and regions with

618 low scores (bottom 5\%) or no scores because the corresponding genes were not indexed by MyTaxa

619 were excluded from further analysis (filtered genome). Note that detection limit for the filtered genome

620 (dashed line) is lower than the unfiltered genome (solid line).

621

622 Figure 5. Detection limits when co-occurring relatives are present. Negative and positive examples 623 were constructed using default imGLAD settings except that closely related (non-target) genomes to the 624 target genome at varied levels of ANI (90-100\%) were included in the datasets. Results shown are based 625 on E. coli - E. albertii genomes related to the target E. coli O157-H7 strain. The values shown (y-axis) 626 represent the McFadden's pseudo- $R^{2}$ measure of the confidence level of the regression parameters of 627 the logistic function by comparing the fitted model estimated by imGLAD to a standard, null model that 628 only contains an intercept variable (see Materials and Methods for further details). Note that the model 629 performs reasonably well $\left(R^{2}>0.7\right)$ until the training set shows $\sim 98.5 \%$ ANI relatedness at which point 630 the pseudo- $R^{2}$ falls near 0.5 or below, indicating that the logistic function was not robust.

631

632

633 


\section{REFERENCES}

635

636

637

638

639

640

641

642

643

644

645

646

647

648

649

650

651

652

653

654

655

656

657

658

659

660

661

662

663

664

665

666

667

668

Ahn TH, Chai J, and Pan C. 2015. Sigma: strain-level inference of genomes from metagenomic analysis for biosurveillance. Bioinformatics 31:170-177. 10.1093/bioinformatics/btu641

Altschul SF, Madden TL, Schaffer AA, Zhang J, Zhang Z, Miller W, and Lipman DJ. 1997. Gapped BLAST and PSI-BLAST: a new generation of protein database search programs. Nucl Acids Res 25:33893402.

Be NA, Thissen JB, Gardner SN, McLoughlin KS, Fofanov VY, Koshinsky H, Ellingson SR, Brettin TS, Jackson $\mathrm{PJ}$, and Jaing CJ. 2013. Detection of Bacillus anthracis DNA in complex soil and air samples using next-generation sequencing. PLOS ONE 8:e73455. 10.1371/journal.pone.0073455

Caro-Quintero A, and Konstantinidis KT. 2012. Bacterial species may exist, metagenomics reveal. Environmental Microbiology 14:347-355. 10.1111/j.1462-2920.2011.02668.x

Carter MQ, Brandl MT, Louie JW, Kyle JL, Carychao DK, Cooley MB, Parker CT, Bates AH, and Mandrell RE. 2011. Distinct acid resistance and survival fitness displayed by Curli variants of enterohemorrhagic Escherichia coli 0157:H7. Appl Environ Microbiol 77:3685-3695. 10.1128/AEM.02315-10

Freitas TA, Li PE, Scholz MB, and Chain PS. 2015. Accurate read-based metagenome characterization using a hierarchical suite of unique signatures. Nucleic acids research 43:e69. 10.1093/nar/gkv180

Goris J, Konstantinidis KT, Klappenbach JA, Coenye T, Vandamme P, and Tiedje JM. 2007. DNA-DNA hybridization values and their relationship to whole-genome sequence similarities. International journal of systematic and evolutionary microbiology 57:81-91. 57/1/81 [pii]

10.1099/ijs.0.64483-0

Hong C, Manimaran S, Shen Y, Perez-Rogers JF, Byrd AL, Castro-Nallar E, Crandall KA, and Johnson WE. 2014. PathoScope 2.0: a complete computational framework for strain identification in environmental or clinical sequencing samples. Microbiome 2:33. 10.1186/2049-2618-2-33

Huang AD, Luo C, Pena-Gonzalez A, Weigand MR, Tarr CL, and Konstantinidis KT. 2017. Metagenomics of Two Severe Foodborne Outbreaks Provides Diagnostic Signatures and Signs of Coinfection Not Attainable by Traditional Methods. Appl Environ Microbiol 83. 10.1128/AEM.02577-16

Huang W, Li L, Myers JR, and Marth GT. 2012. ART: a next-generation sequencing read simulator. Bioinformatics 28:593-594. 10.1093/bioinformatics/btr708

Jones E, Oliphant TE, and Peterson P. 2001. SciPy: Open Source Scientific Tools for Python. http://wwwscipyorg/.

Kent WJ. 2002. BLAT--the BLAST-like alignment tool. Genome research 12:656-664. 10.1101/gr.229202. Article published online before March 2002 
669

670

671

672

673

674

675

676

677

678

679

680

681

682

683

684

685

686

687

688

689

690

691

692

693

694

695

696

697

698

699

700

701

702

703

Konstantinidis KT, and Tiedje JM. 2005. Genomic insights that advance the species definition for prokaryotes. Proc Natl Acad Sci U S A 102:2567-2572.

Langmead B, and Salzberg SL. 2012. Fast gapped-read alignment with Bowtie 2. Nat Methods 9:357-359. 10.1038/nmeth.1923

Luo C, Knight R, Siljander H, Knip M, Xavier RJ, and Gevers D. 2015. ConStrains identifies microbial strains in metagenomic datasets. Nat Biotechnol 33:1045-1052. 10.1038/nbt.3319

Luo C, Rodriguez RL, and Konstantinidis KT. 2014a. MyTaxa: an advanced taxonomic classifier for genomic and metagenomic sequences. Nucleic acids research 42:e73. 10.1093/nar/gku169

Luo C, Rodriguez-R LM, Johnston ER, Wu L, Cheng L, Xue K, Tu Q, Deng Y, He Z, Shi JZ, Yuan MM, Sherry RA, Li D, Luo Y, Schuur EAG, Chain P, Tiedje JM, Zhou J, and Konstantinidis KT. 2014b. Soil Microbial Community Responses to a Decade of Warming as Revealed by Comparative Metagenomics. Applied and Environmental Microbiology 80:1777-1786. 10.1128/aem.03712-13

Oliphant TE. 2006. A guide to NumPy. USA: Tregol Publishing.

Pruitt KD, Tatusova T, and Maglott DR. 2007. NCBI reference sequences (RefSeq): a curated nonredundant sequence database of genomes, transcripts and proteins. Nucleic acids research 35:D61-65. 10.1093/nar/gkl842

Rodriguez RL, Castro JC, Kyrpides NC, Cole JR, Tiedje JM, and Konstantinidis KT. 2018. How Much Do rRNA Gene Surveys Underestimate Extant Bacterial Diversity? Appl Environ Microbiol 84. 10.1128/AEM.00014-18

Segata N, Waldron L, Ballarini A, Narasimhan V, Jousson O, and Huttenhower C. 2012. Metagenomic microbial community profiling using unique clade-specific marker genes. Nat Meth 9:811-814. http://www.nature.com/nmeth/journal/v9/n8/abs/nmeth.2066.html - supplementaryinformation

Sharma MK, Narayanan J, Pardasani D, Srivastava DN, Upadhyay S, and Goel AK. 2016. Ultrasensitive electrochemical immunoassay for surface array protein, a Bacillus anthracis biomarker using AuPd nanocrystals loaded on boron-nitride nanosheets as catalytic labels. Biosens Bioelectron 80:442-449. 10.1016/j.bios.2016.02.008

Skipper S, and Perktold J. 2010. Statsmodels: Econometric and statistical modeling with python. . Proceedings of the 9th Python in Science Conference.

Truong DT, Franzosa EA, Tickle TL, Scholz M, Weingart G, Pasolli E, Tett A, Huttenhower C, and Segata N. 2015. MetaPhIAn2 for enhanced metagenomic taxonomic profiling. Nat Methods 12:902-903. 10.1038/nmeth.3589

WendI MC, Kota K, Weinstock GM, and Mitreva M. 2012. Coverage theories for metagenomic DNA sequencing based on a generalization of Stevens' theorem. J Math Biol. 10.1007/s00285-0120586-x 
704 Zolfo M, Tett A, Jousson O, Donati C, and Segata N. 2017. MetaMLST: multi-locus strain-level bacterial 


\section{Figure 1 (on next page)}

Schematic representation of imGLAD's pipeline.

imGLAD has two main components. (A) The first part (training) consists of a learning procedure, in which a set of in-silico generated datasets are fitted through a logistic model that aims to separate positive from negative datasets. For this, a database of 200 genomes is used to generate the simulated Illumina reads of these datasets. Reads simulated from the target genome are then incorporated into half of the simulated datasets. The resulting datasets are marked as positive for training while the other half is marked as negative. Sequencing depth and breadth of the target (reference) genome are calculated for each dataset. A logistic function is then fitted to the data to separate positive from negative examples. The regression parameters are stored for further use. (B) The second part (estimation) consists of estimating the sequencing breadth and/or depth values of the target genome provided by the (recruited) reads of the experimental metagenomes, and comparison of the derived sequencing depth and breadth values to those of the logistic function from the training step. 


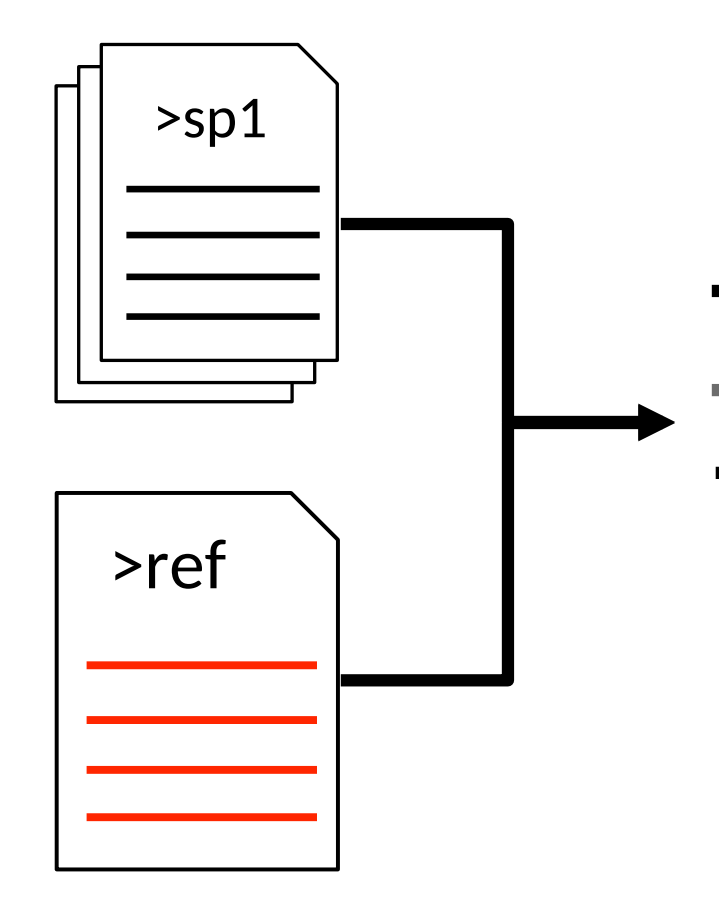

Illumina
reads
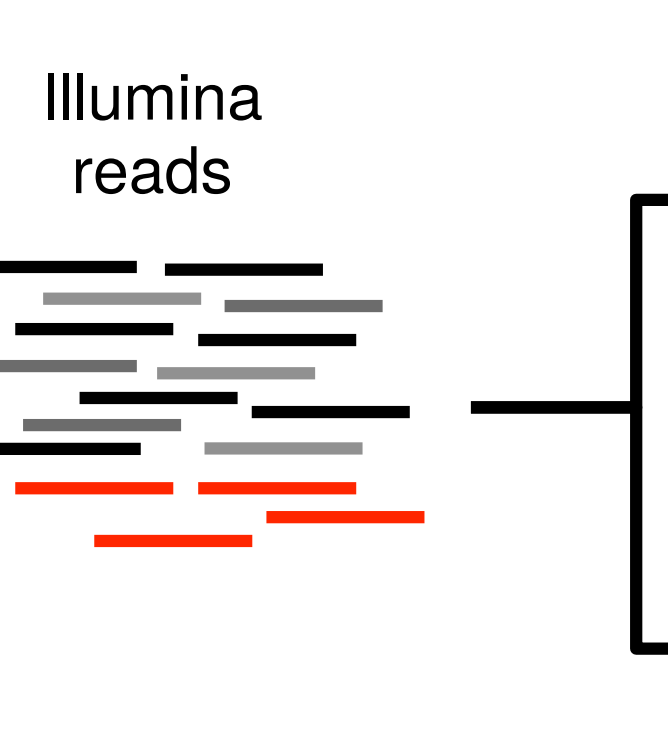

Negative metagenomes
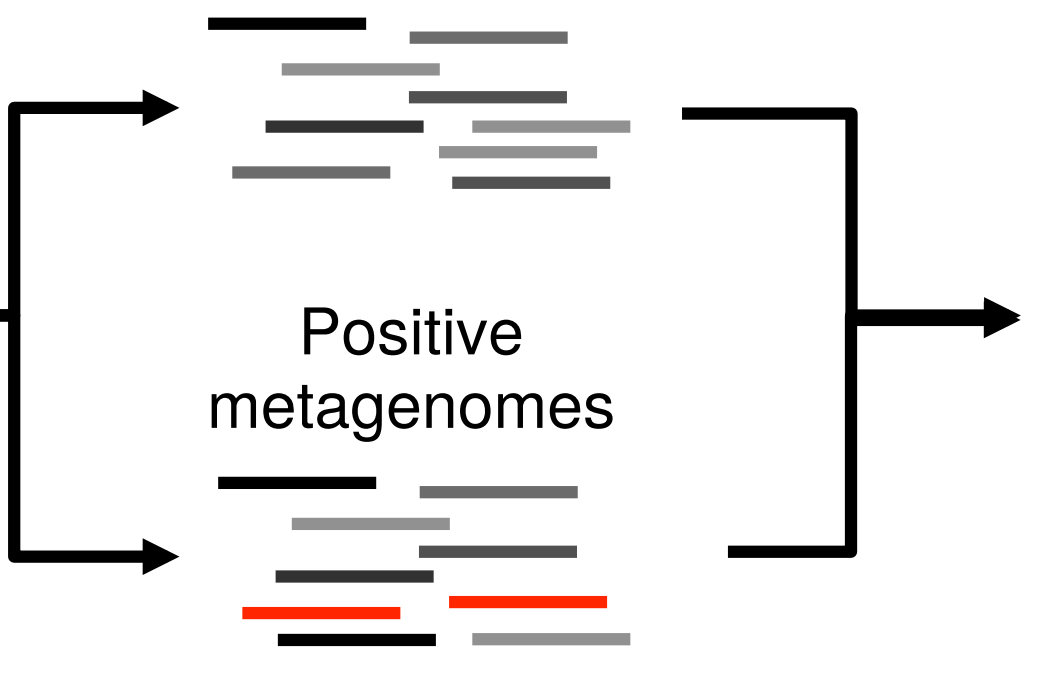

Read mapping

Model training

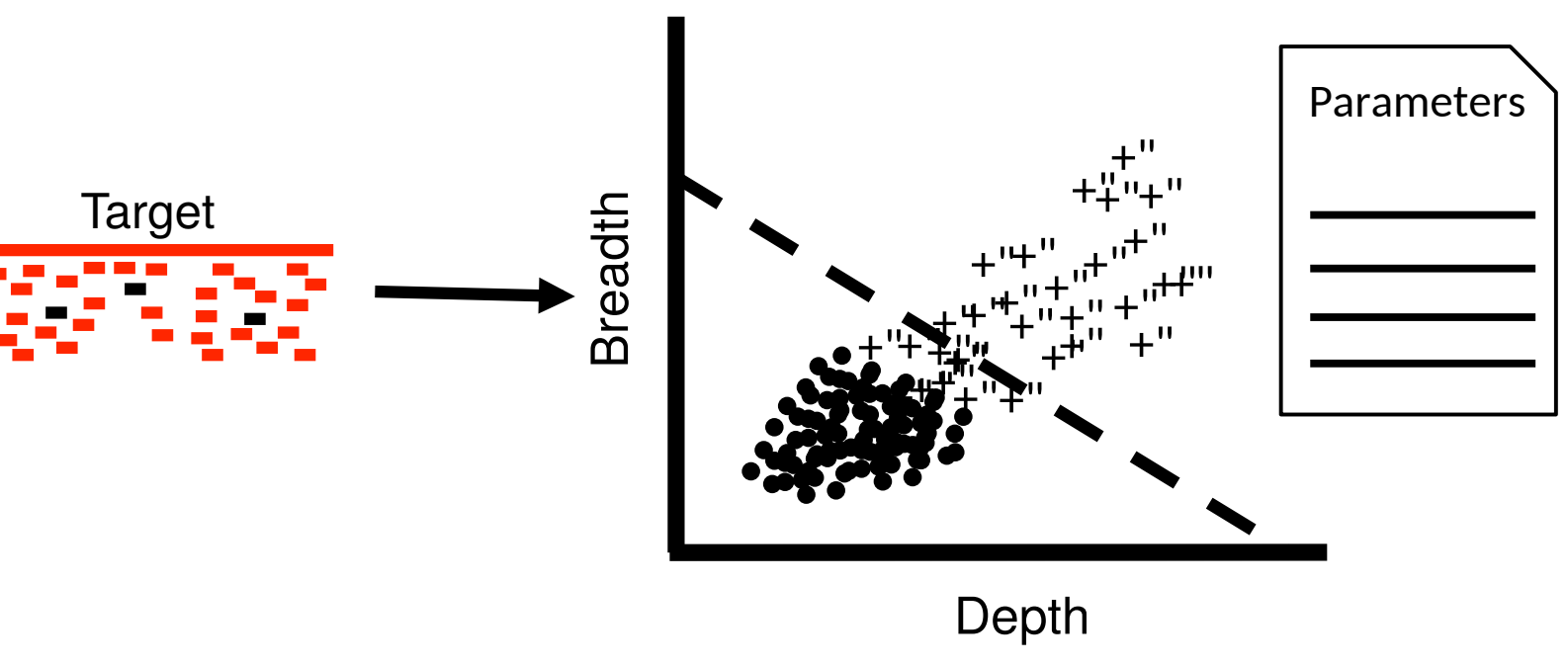

Read mapping genome -

Filter

Filtered genome

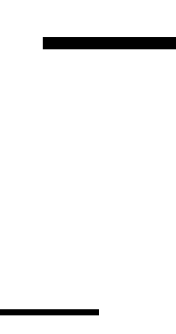

Metagenomic reads

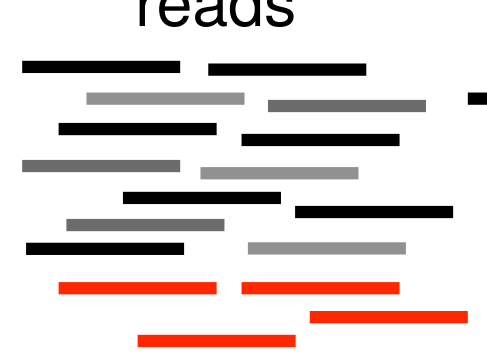

$-$

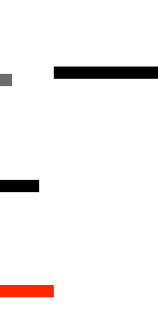

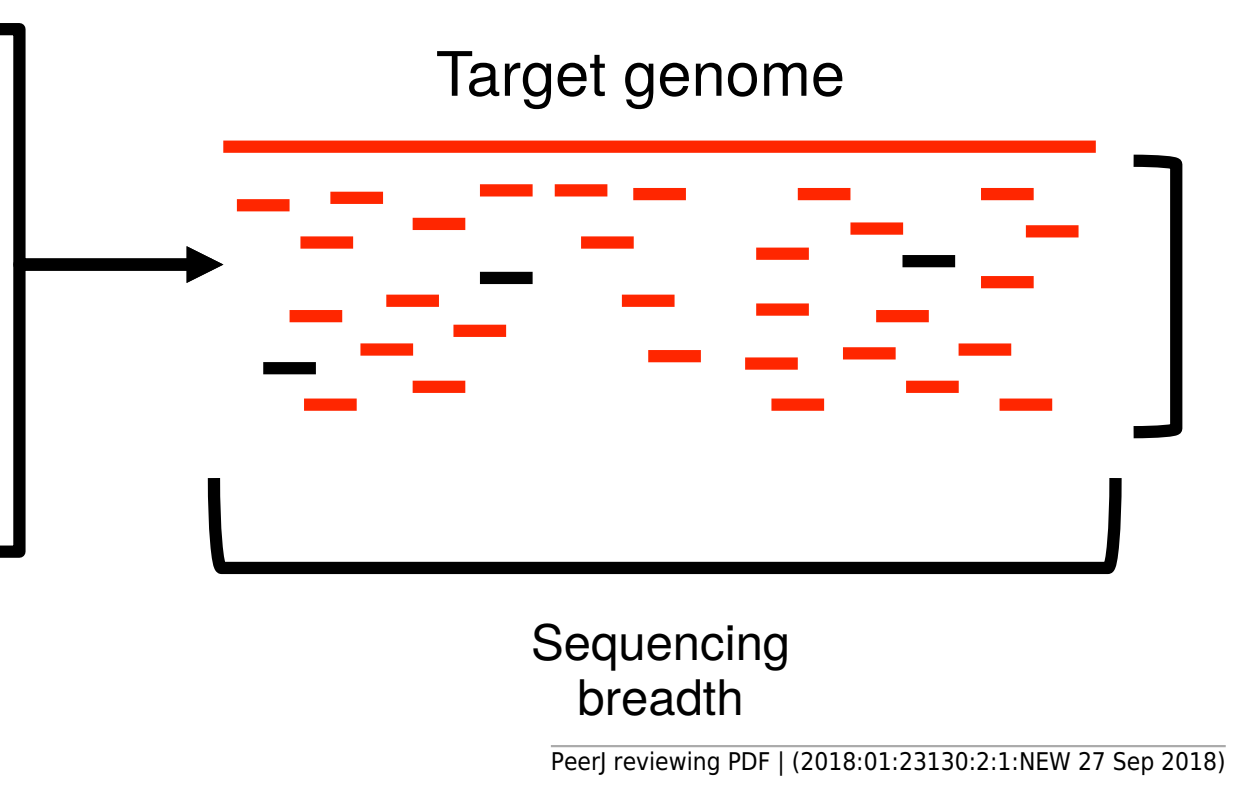

Sequencing depth

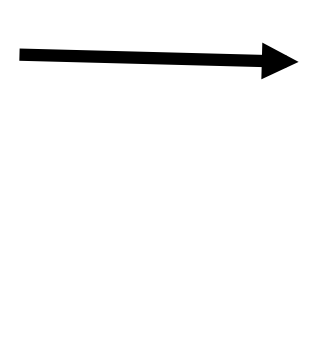

Peer) reviewing PDF | (2018:01:23130:2:1:NEW 27 Sep 2018) 


\section{Figure 2}

Identification of target genomes in metagenomic datasets with imGLAD.

Positive datasets (crosses) are separated form negative datasets (dots) through a logistic function (solid line) based on in-silico training datasets. (A) Datasets with reads of $E$. coli are separated form negative datasets. (B) Datasets with reads of $B$. anthracis are separated form negative datasets. Red asterisks denote the position of the experimental metagenomes (remaining dots represent in-silico generated datasets). Note the differences in scale on the $x$-axes between positive and negative datasets. 

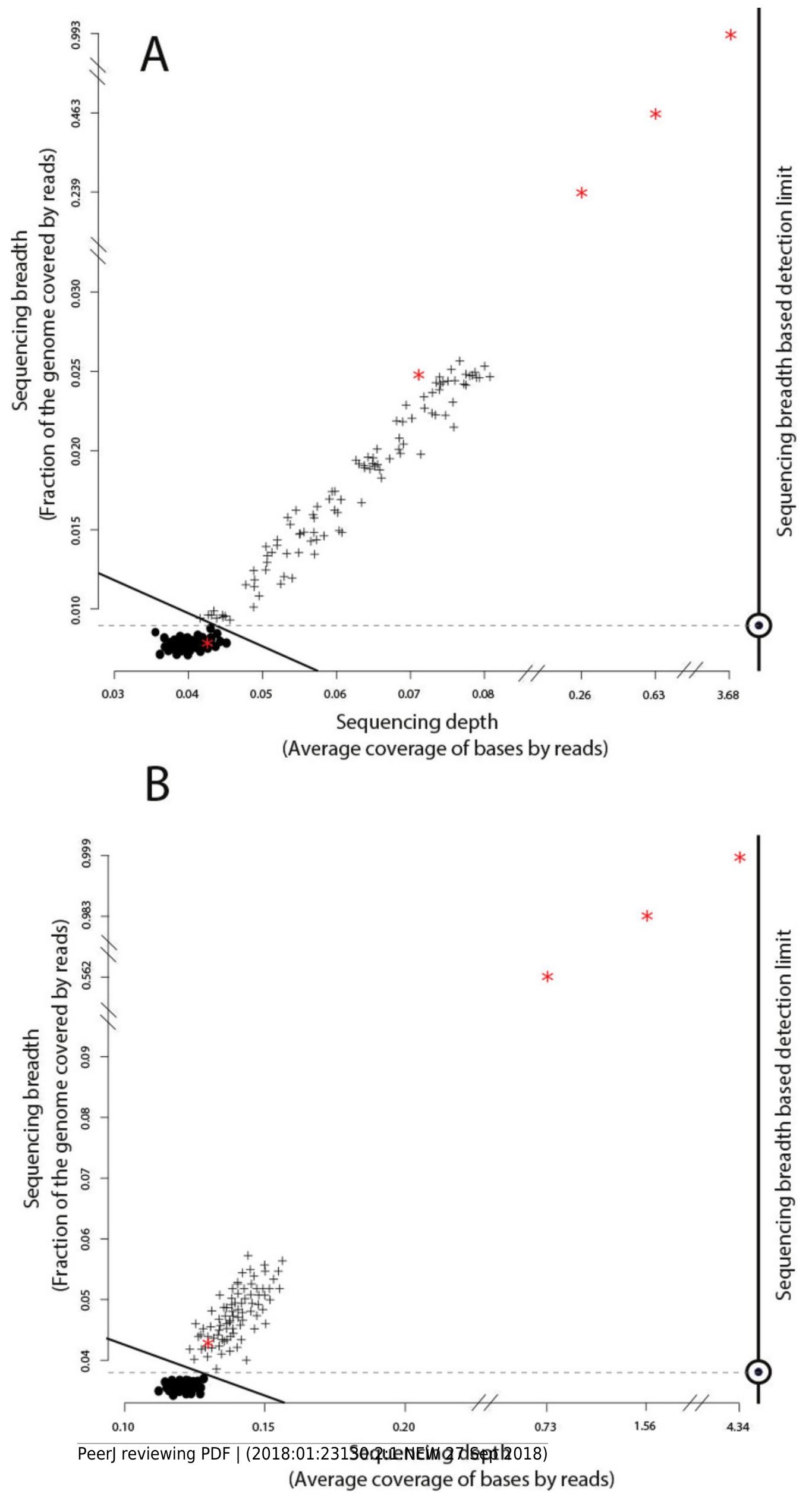


\section{Figure 3 (on next page)}

Performance of imGLAD in comparison to Pathoscope, MetaPhIAn and MetaMLST.

(A) in-silico synthesized datasets from 200 RefSeq genomes were spiked with E. coli EC11 reads at different abundances (reflected by sequencing breadth, $x$-axes) to test the sensitivity of imGLAD (y-axes), i.e., the proportion of properly classified positive datasets among the total number of positive datasets. (B) Similar comparisons based on 571 datasets from the HMP project, which did not contain any E. coli signal and were spiked with different concentrations of $E$. coli EC11 reads. (C) imGLAD was evaluated in the same datasets as in panel A but this time the datasets included, in addition to the RefSeq genomes, $10 \mathrm{E}$. coli genomes with ANI ranging between $95-98 \%$ to the target $E$. coli EC11 genome spiked in at the same concentration (i.e., $0.3 \mathrm{X}$ ). Note that as sequencing breadth increases the sensitivity of the prediction is higher for all tools tested, with the exception of MetaMLST that requires at least $2 X$ sequencing depth for robust detection (see text for details). However, imGLAD can effectively classify samples at 100\% sensitivity (as positive samples in this case) with a sequencing breadth as low as 0.03 (i.e., $3 \%$ of the target genome recovered) or less, whereas the other tools show lower sensitivity at these levels in all cases evaluated. 

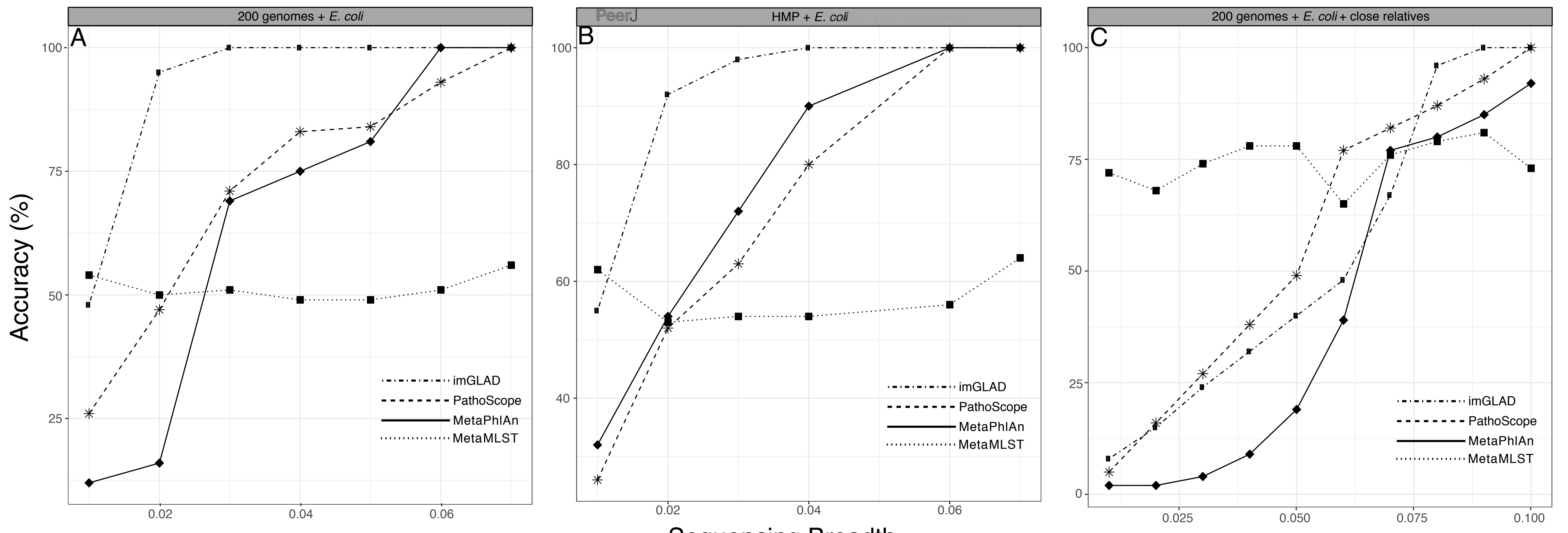
Sequencing Breadth 
Figure 4 (on next page)

Effect of filtering of less informative genes by MyTaxa on minimum sequencing breadth and depth.

Genome regions of the $E$. coli target genome were classified by MyTaxa, and regions with low scores (bottom 5\%) or no scores because the corresponding genes were not indexed by MyTaxa were excluded from further analysis (filtered genome). Note that detection limit for the filtered genome (dashed line) is lower than the unfiltered genome (solid line). 


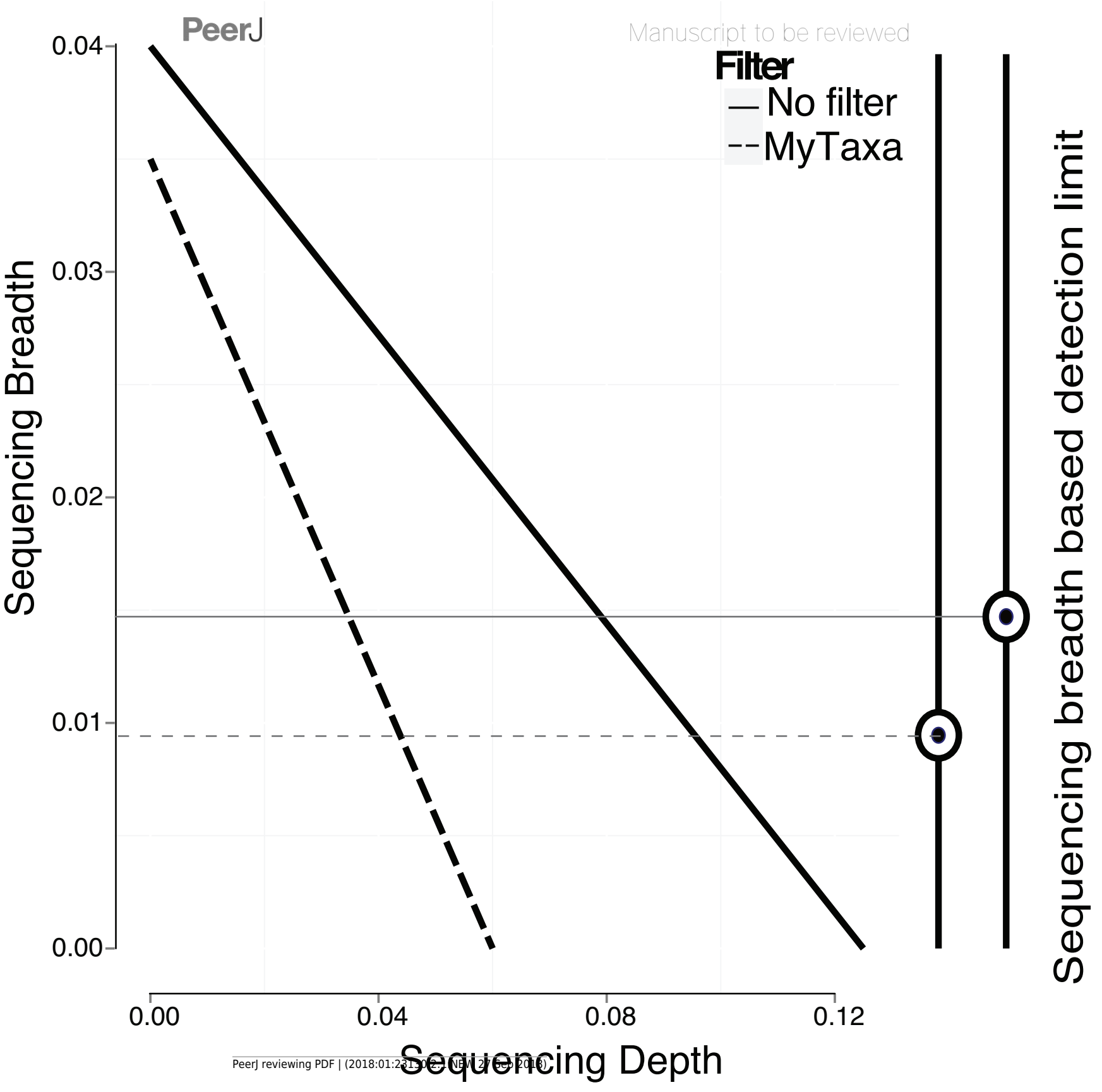




\section{Figure $\mathbf{5}$ (on next page)}

Detection limits when co-occurring relatives are present.

Negative and positive examples were constructed using default imGLAD settings except that closely related (non-target) genomes to the target genome at varied levels of ANI (90-100\%) were included in the datasets. Results shown are based on E. coli - E. albertii genomes related to the target E. coli 0157-H7 strain. The values shown (y-axis) represent the McFadden's pseudo- $\mathrm{R}^{2}$ measure of the confidence level of the regression parameters of the logistic function by comparing the fitted model estimated by imGLAD to a standard, null model that only contains an intercept variable (see Materials and Methods for further details). Note that the model performs reasonably well $\left(R^{2}>0.7\right)$ until the training set shows $\sim 98.5 \%$ ANI relatedness at which point the pseudo- $R^{2}$ falls near 0.5 or below, indicating that the logistic function was not robust. 


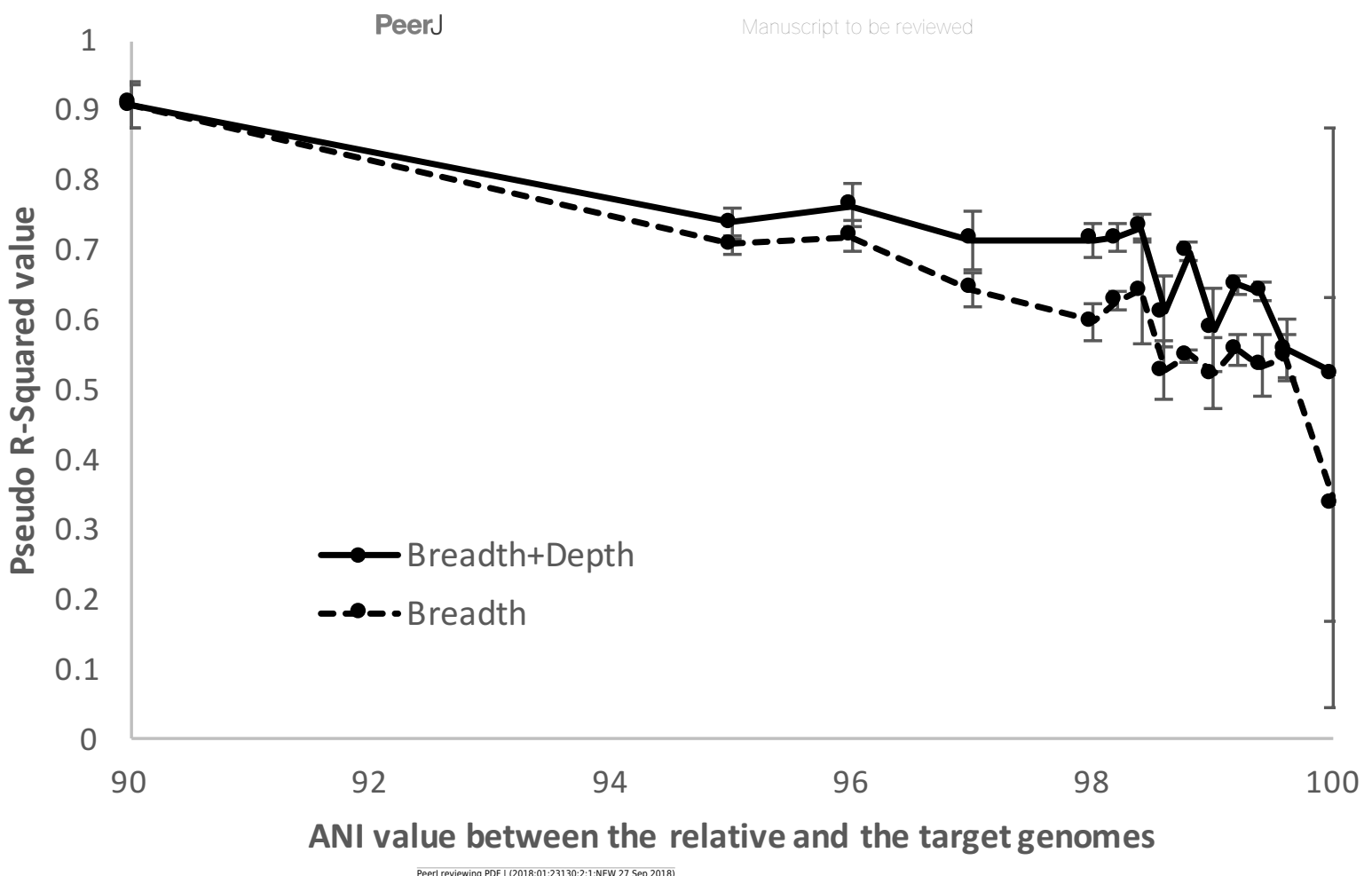




\section{Table $\mathbf{1}$ (on next page)}

Samples inoculated with different cell concentrations of E. coli ( $1^{\text {st }}$ column) were classified by imGLAD as present/positive or absent/negative.

The calculated breadth of the $E$. coli reference genome recovered ( $\left({ }^{\text {nd }}\right.$ column $)$ and the sequencing depth ( $3^{\text {rd }}$ column) as well as the derived probability of presence ( $4^{\text {th }}$ column) are shown. The estimated relative abundance (fraction of total cells) of $E$. coli in the sample is also shown ( $6^{\text {th }}$ column). Relative abundance was estimated based on the number of reads mapping on the $E$. coli genome after filtering relative to the total number of reads of the metagenome, assuming all community members had an average genome size of $5 \mathrm{Mbp}$ (similar to E. coli genome size) and all mapped reads originated from the $E$. coli cells spiked in ( $1^{\text {st }}$ column) without losses, i.e., relative abundance $=$ fraction of total reads mapping to $E$. coli. All samples were found positive for presence of $E$. coli $(p$-value $=0.004)$ except the control sample without inoculated $E$. coli cells. 
1 Table 1. Samples inoculated with different cell concentrations of $E$. coli (1 ${ }^{\text {st }}$ column) were 2 classified by imGLAD as present/positive or absent/negative.

\begin{tabular}{crcrrc}
\hline Sample & $\begin{array}{c}\text { Sequencing } \\
\text { breadth }\end{array}$ & Sequencing depth & $\begin{array}{c}\text { E. coli presence } \\
\text { (p-value) }\end{array}$ & $\begin{array}{c}\text { Library size } \\
\text { (in Mbp) }\end{array}$ & $\begin{array}{c}\text { E. coli relative } \\
\text { abundance }\end{array}$ \\
\hline Control & 0.007 & 0.042 & 0.847 & $4,664,749$ & ND \\
80 cells & 0.239 & 0.262 & 0.004 & $4,957,122$ & $2.44 \mathrm{E}-05$ \\
$8 \times 10^{3}$ cells & 0.463 & 0.639 & $7.1 \times 10^{-4}$ & $3,895,441$ & 0.0033 \\
$8 \times 10^{5}$ cells & 0.993 & 3.683 & $1 \times 10^{-5}$ & $3,705,361$ & 0.3463
\end{tabular}

3 The calculated breadth of the E. coli reference genome recovered ( $2^{\text {nd }}$ column) and the sequencing depth 4 ( $3^{\text {rd }}$ column) as well as the derived probability of presence ( $4^{\text {th }}$ column) are shown. The estimated relative 5 abundance (fraction of total cells) of $E$. coli in the sample is also shown (6 $6^{\text {th }}$ column). Relative abundance 6 was estimated based on the number of reads mapping on the $E$. coli genome after filtering relative to the 7 total number of reads of the metagenome, assuming all community members had an average genome 8 size of $5 \mathrm{Mbp}$ (similar to $E$. coli genome size) and all mapped reads originated from the $E$. coli cells spiked 9 in ( $1^{\text {st }}$ column) without losses, i.e., relative abundance $=$ fraction of total reads mapping to $E$. coli. All 10 samples were found positive for presence of $E$. coli $(p$-value $=0.004)$ except the control sample without 11 inoculated E. coli cells. 


\section{Table 2 (on next page)}

Soil samples inoculated with different copies of $B$. anthracis strain Ames genomic DNA ( $1^{\text {st }}$ column) were classified by imGLAD as present/positive or absent/negative.

The calculated breadth of the $B$. anthracis reference genome recovered $\left(2^{\text {nd }}\right.$ column $)$ and the sequencing depth ( $3^{\text {rd }}$ column) as well as the derived probability of presence ( $4^{\text {th }}$ column) are shown. Samples with a number of genome higher or equal to 100 genomes were classified as positive samples. Samples with 1 and 10 genomic copies were indistinguishable from the negative samples of the training set. 
1 Table 2. Soil samples inoculated with different copies of $B$. anthracis strain Ames genomic DNA 2 ( $1^{\text {st }}$ column) were classified by imGLAD as present/positive or absent/negative.

\begin{tabular}{cccc}
\hline Sample & Sequencing breadth & Sequencing depth & $\begin{array}{c}\text { B. anthracis presence } \\
\text { (p-value) }\end{array}$ \\
\hline 1 Genome & $1.56 \times 10^{-3}$ & $2.0 \times 10^{-3}$ & 0.999 \\
10 Genomes & 0.001 & 0.003 & 0.998 \\
100 Genomes & 0.039 & 0.128 & 0.002 \\
$10^{3}$ Genomes & 0.562 & 0.732 & $1.4 \times 10^{-3}$ \\
$10^{4}$ Genomes & 0.983 & 1.563 & 0 \\
$10^{5}$ Genomes & 0.999 & 4.34 & 0
\end{tabular}

3 The calculated breadth of the $B$. anthracis reference genome recovered ( $2^{\text {nd }}$ column) and the sequencing 4 depth ( $3^{\text {rd }}$ column) as well as the derived probability of presence ( $4^{\text {th }}$ column) are shown. Samples with a 5 number of genome higher or equal to 100 genomes were classified as positive samples. Samples with 1 6 and 10 genomic copies were indistinguishable from the negative samples of the training set. 